\title{
Deficits in Response Space Following Unilateral Striatal Dopamine Depletion in the Rat
}

\author{
Verity J. Brown and Trevor W. Robbins \\ Department of Experimental Psychology, University of Cambridge, Cambridge CB2 3EB, United Kingdom
}

\begin{abstract}
Hungry rats were trained to report the occurrence and location of brief, unpredictable visual stimuli presented to the left of their heads in 1 of 2 response locations. After training, they received unilateral infusions of 6-hydroxydopamine, depleting dopamine throughout the head of the caudate putamen, either on the left or the right side, that is, either ipsilateral or contralateral to the side on which they were required to respond.

Following an ipsilateral lesion there were no impairments in localization of the visual discriminanda and there was no lengthening of reaction time. The contralaterally lesioned rats, however, showed considerably lengthened reaction times to both stimuli and a profound bias to the nearer of the 2 response locations.

Evidence from probe trials in which the visual stimuli were presented separately or simultaneously showed that the impairment was not due to a failure to localize the stimuli in contralateral space but, rather, resulted from a deficit in directing responses in contralateral space. It is suggested that this may be due to a distortion in the representation of response space.
\end{abstract}

Unilateral striatal dopamine (DA) depletion results in a polymodal "neglect" characterized by a failure to orient to contralateral stimuli (Marshall et al., 1971, 1980; Marshall and Teitelbaum, 1974; Ljungberg and Ungerstedt, 1976; Dunnett and Iversen, 1982; Fairley and Marshall, 1986). The neglect is not primarily sensory in nature but is evident only when a contralateral response is required (Carli et al., 1985). Although Carli et al. showed that rats with unilateral striatal DA depletion can detect contralateral events, they did not rule out the possibility of a hemispatial disability; the rat may be unable to localize sensory events occurring in the neglected hemispace. It has been suggested that the reaction time deficits found following bilateral striatal DA depletion in rats could reflect an impairment in the processing of sensory information necessary to initiate a response (Amalric and Koob, 1987), and thus even an apparently pure output type deficit could have a sensory component.

In the present experiments, we tested whether striatal DA depletion impaired the ability to localize stimuli accurately in contralateral space by training rats to discriminate the spatial

\footnotetext{
Received June 15, 1988; revised Aug. 8, 1988; accepted Aug. 23, 1988.

This work was supported by the Parkinson's Disease Society (UK). We would like to thank Dr. J. Downes for his helpful comments on the manuscript, and G. H. Jones for technical assistance with the biochemistry. V.J.B. is in receipt of a Medical Research Council (UK) studentship.

Correspondence should be addressed to Verity J. Brown, Department of Experimental Psychology, University of Cambridge, Downing Street, Cambridge CB2 3EB, UK

Copyright $\subset 1989$ Society for Neuroscience $0270-6474 / 89 / 030983-07 \$ 02.00 / 0$
}

location of stimuli on one side of space only, before depleting DA in the striatum contralateral to that side. In another group of rats, DA was depleted from the striatum on the same side as the discrimination, as a control procedure for nonspecific postoperative effects.

A second question of importance concerning striatal neglect is whether a possible hemispatial deficit induced by unilateral striatal lesions is restricted to one side of the body or, alternatively, is relative, or allocentric, in nature. Kinsbourne and Warrington (1962) and Bisiach and Luzzatti (1978) found parietal neglect not only for left retinotopic, but also for left conceptual space. In a human reaction time task, Ladavas (1987) described 2 components of the neglect; there was not only an overall lengthening of reaction times to stimuli in the left, neglected, visual field, but also a reduced reactivity to stimuli occupying a relatively leftwards position in either visual field.

A similar distinction might be appropriate in an analysis of striatal neglect in the rat. The present cxpcrimental design allows 2 tests to be made of this hypothesis. First, rats with contralateral lesions should be biased towards the nearer of the 2 response locations; that is, relative ipsilateral space. Second, rats with ipsilateral lesions should be biased away from the nearer response location, making more responses in the far, relatively ipsilateral, response hole.

\section{Materials and Methods}

\section{Animals}

Sixteen male Lister Hooded rats (Olac, Bicester), weighing 200-250 gm at the beginning of the experiment, were housed in pairs, in a temperature-regulated $\left(22^{\circ} \mathrm{C}\right)$ environment in natural daylight. They were maintained at $90 \%$ of their free-feeding body weight throughout the experiment, on $15 \mathrm{gm} / \mathrm{d} / \mathrm{rat}$ of standard laboratory chow, provided after the daily experimental session.

\section{Surgery}

When stable preoperative performance had been reached, the rats received unilateral lesions of the caudate putamen, by infusion of the neurotoxin 6-hydroxydopamine (6-OHD $\Lambda$ ).

The rats were treated with $50 \mathrm{mg} / \mathrm{kg}$ of pargyline hydrochloride (Sigma Chemical Co.) dissolved in $0.9 \%$ sterile saline, injected i.p. $30 \mathrm{~min}$ prior to surgery, to enhance the effect of 6-OHDA (Breese and Traylor, 1971). They were then anesthetized with Equithesin $(0.3 \mathrm{ml} / 100 \mathrm{gm})$ and unilaterally injected with $2 \mu \mathrm{l}$ of vehicle $(0.1 \mathrm{mg} / \mathrm{ml}$ ascorbic acid in $0.9 \%$ saline) containing $8 \mu \mathrm{g}$ of 6-OHDA base (6-OHDA hydrobromide, Sigma Chemical Co.) via a 30-gauge stainless steel cannula at the following placement: anterior $+2.0 \mathrm{~mm}$ from bregma; lateral $\pm 3.5 \mathrm{~mm}$ from midline; dorsal $-5.5 \mathrm{~mm}$ from cortical surface, in the plane of Pellegrino et al. (1979). The cannula was left in place for a further 2 $\mathrm{min}$ before and after the infusion.

\section{Neurochemical analysis}

Six weeks after surgery, the rats were killed under ether anesthesia, and the brains rapidly removed. The tissue was dissected on ice as previously 


Sustcined nose poke

$$
\begin{aligned}
& \text { photocell beam } \\
& \text { stimulus }
\end{aligned}
$$

Figure 1. Requirements of the task. A brief $(0.20 \mathrm{sec})$, temporally unpredictable, visual stimulus was presented in 1 of 2 locations to the left of the rat's head. A correct response was recorded when the rat interrupted the photocell beam in the stimulus location. An incorrect response was recorded for responses in the unlit hole.

described (Koob et al., 1978) into the nucleus accumbens, the anterior caudate putamen (taken from the same 2-mm-thick slice), and the posterior caudate putamen and the tail of caudate, taken from the next two $2 \mathrm{~mm}$ slices immediately posterior. The tissue was stored at $-70^{\circ} \mathrm{C}$ until sampled.

Tissue concentrations of DA were measured by high-performance liquid chromatography with electrochemical detection (HPLC-EC) based on the method described by Mefford (1981).

\section{Apparatus}

Three aluminum chambers $(26 \times 26 \times 26 \mathrm{~cm})$ were used. Set into the curved rear wall of each chamber were 9,4 -cm-deep holes $(2.5 \times 2.5$ $\mathrm{cm})$. The holes were arranged in a horizontal radiating array, $2 \mathrm{~cm}$ above the grid floor (see figure 1 of Carli et al., 1983). In the present experiment, only the hole central in the array, and the 2 holes adjacent on the left were open and in use; the others were blocked with metal caps. Across the entrance of each hole was a vertically directed infrared photocell beam. The stimuli were presented by the illumination of a $2.8 \mathrm{~W}$ bulb at the rear of each hole. Nonreflective gray paint on the inside of the chambers minimized the possibility that the rat could be detecting the stimuli by reflections on the walls rather than from its source in the hole. On the front wall of the chamber was a food magazine, covered by a hinged Plexiglas panel.

Each chamber was controlled and the data collected online by a microcomputer (Control Universal, U.K.).

\section{Procedure}

Training. Initially, all the holes were blocked, and the rats were trained to collect pellets $(45 \mathrm{mg}$, Dustless precision pellets, BioServ Inc., New Jersey) delivered under a fixed time, $30 \mathrm{sec}$ schedule (FT30).

In a further 3 sessions, the central hole of the array was unblocked and illuminated, and the rats were rewarded on a continuous reinforcement schedule for interrupting the photocell beam in the hole.

The caps were then removed from the 2 side holes on the left of the central hole. Pushing the panel in front of the food magazine initiated the trial and the central hole was illuminated. A response in the illuminated hole extinguished that light and began one of 4 intervals (delays) $-0,0.5,1.0$ or $1.5 \mathrm{sec}$-culminating in the presentation of the brief $(0.20 \mathrm{sec})$ visual stimulus in either one of the side holes. The size of the hole ensured that the rat, to break the photocell beam, was oriented forwards and, therefore, that the stimuli were presented to its left eye only. The intention was not to restrict visual input to one hemisphere only (in the rat's visual system about $25 \%$ of the fibers are crossed
(Cowey and Francini, 1979); therefore, it can be assumed that the information was available in both hemispheres), although previous experiments have demonstrated that stimuli presented to the left side are not detected by the right eye (Mittleman et al., 1988). The depth of the holes meant that the stimulus light was not perceived as a point source, but shone diffusely from each hole. The rat was required to report the occurrence and the location of the light by withdrawing its nose from the central hole and responding in the hole where the stimulus had occurred. Figure 1 shows these task requirements.

A correct response resulted in the delivery of a food pellet. Incorrect or premature responses were punished with $1 \mathrm{sec}$ of darkness (time out). All rats were trained to respond to the left; in previous experiments in the same apparatus, no evidence had been found to suggest that there might be a population asymmetry in the performance of this task or that unilateral striatal DA depletion produces such a population asymmetry (M. Carli, J. L. Evenden, and T. W. Robbins, unpublished observations).

Baseline performance. Each daily session consisted of 80 trials, with 10 trials of each of the 4 delays, in each of the 2 response holes, presented in a pseudo-random order. The rats were tested for 5 consecutive days to obtain the preoperative baseline values, and $5 \mathrm{~d}$ after a period of postoperative recovery lasting $4 \mathrm{~d}$. They were given a second period of postoperative testing $30 \mathrm{~d}$ after surgery.

Effects of manipulating the visual stimuli. Postoperatively, the rats were tested on probe trials in which either both the stimuli were presented simultaneously, in a test analogous to the clinical test for sensory extinction (Bender, 1952), as a means of possibly exacerbating the deficits, or neither stimulus was presented, so as to assess the effect on behavior when no stimulus is detected. In addition, 2 sessions were given when the stimulus light of one of the response holes was removed. Even when the animals do not see a stimulus, they nevertheless eventually make a response, after having waited for the stimulus to occur. The effects of removing one of the stimuli would therefore be to lengthen reaction times of responses made to that location. By comparing performance under these conditions with that in the baseline condition, it could be ascertained whether any deficits seen in baseline performance could be attributed to an inability to detect one of the stimuli.

Effects of removing response options. To test whether the rats were able to direct responses to both locations postoperatively, 2 sessions were run in which first one and then the other of the 2 left response holes was blocked and the stimuli were presented in one hole only, in what is then a simple reaction time task.

\section{Measures and statistical analysis}

The following variables were analyzed: latency to initiate correct and incorrect responses, taken as the time from the onset of the stimulus to withdrawal from the central hole (reaction time); response bias, measured as the number of correct and incorrect responses in the near left hole divided by total number of responses in both response holes (bias); and frequency of responses made in each of the response holes during periods of timeout (timeout).

The data were subjected to analysis of variance (ANOVA), with surgery as a factor in the analyses. Transformations were applied to normalize the distributions in accordance with the ANOVA model (Winer, 


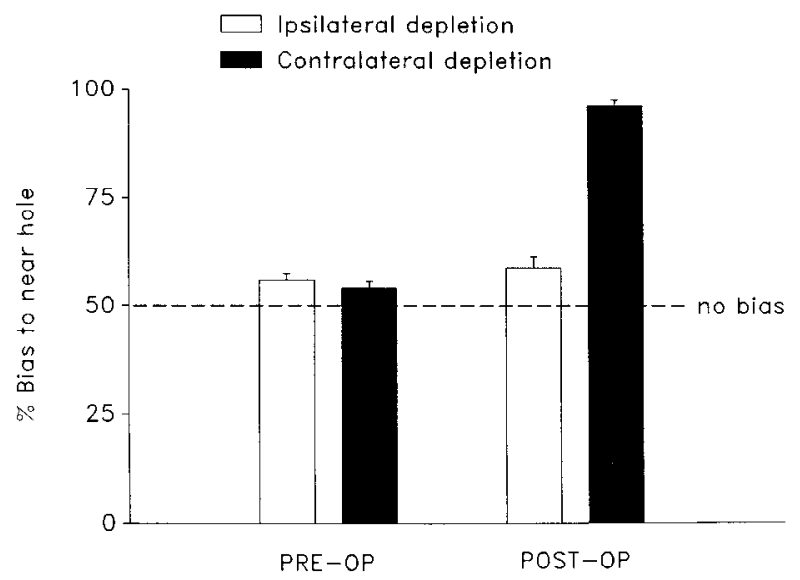

Figure 2. Response bias to the near hole of the ipsilaterally and contralaterally lesioned rats. Bias is (Correct + Incorrect near hole responses)/(Total responses in either hole). Preoperatively, both groups showed a slight bias to the near hole; this remained unchanged in the ipsilaterally lesioned rats, while the contralaterally lesioned rats were significantly more biased.

1971), but as similar results were found, only the untransformed analyses are presented.

\section{Results}

\section{Neurochemical results}

The results of the neurochemical assay of DA concentrations in the different areas of the striatum are shown in Table 1. As can be seen, there was profound $(>90 \%)$ depletion of DA throughout the anterior and posterior caudate putamen compared with the unlesioned side. The tail of the caudate also showed a substantial depletion (70\%), but the nucleus accumbens was depleted by only about $40 \%$.

\section{Response bias}

Figure 2 shows response bias to the near hole pre- and postoperatively for both the lesion groups. Preoperatively, there was a slight bias to the near hole in both groups. This was not significantly increased in the left lesion group; only the right, contralateral lesion group showed an increase in bias, from $54 \%$ preoperatively to $96 \%$ postoperatively. This was reflected in an interaction between the factors of side of Lesion and Surgery $[F(1,15)=157.1, p<0.001]$.

\section{Bias during timeout periods}

The frequency of responses in the near and far holes during periods of timeout, expressed as bias to the near hole, is shown in Figure 3. There was a slight bias to the near hole in both groups preoperatively, which was significantly increased in the contralaterally lesioned animals $[F(1,5)=8.78, p=0.05]$, whereas the ipsilaterally lesioned animals showed a tendency, which approached significance, to be more biased towards the far hole $[F(1,8)=5.06, p=0.055]$.

\section{Reaction time performance}

Figure 4 shows reaction times to the stimuli in the near and far left holes for each lesion group pre- and postoperatively. Preoperative reaction times to the near left hole were faster than those to the far left hole, reflected in a main effect of Hole in an analysis of the preoperative data $[F(1,12)=46.6, p<0.001]$. This pattern remained unchanged in the group lesioned on the

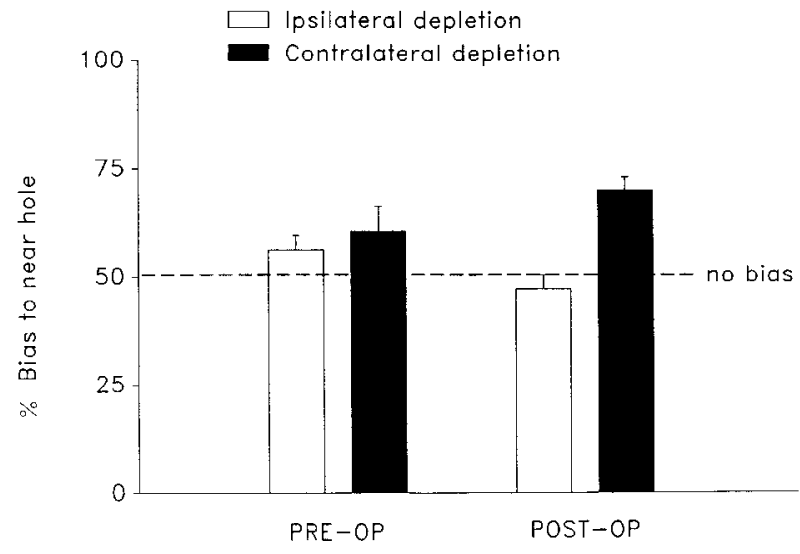

Figure 3. Response bias to the near hole during periods of timeout pre- and postoperatively. Bias was significantly increased in the contralaterally lesioned rats, while the ipsilaterally lesioned animals showed a near-significant tendency to be biased away from the near hole.

left; that is, ipsilateral to the side of the task. In the contralaterally lesioned group, reaction times to both the stimuli were markedly lengthened and there was no difference between reaction time of near and far hole responses. These effects were reflected in interactions between side of Lesion and Surgery $[F(1,13)=95.4, p<0.001]$ and between Lesion, Surgery, and Hole $[F(1,25)=5.2, p=0.03]$.

Figure 5 shows reaction times to stimuli in each of the response holes for the contralaterally lesioned group pre- and postoperatively plotted as a function of stimulus presentation delay. The increase in reaction time was linear, showing no evidence that the rats were waiting for the duration of the longest delay as a strategy to ensure that the stimulus had occurred before initiating their responses (see Carli et al., 1989).

Incorrect reaction times were analyzed for those rats $(n=6)$ showing a large response bias to the near hole. No difference was found between the latencies to initiate correct and incorrect responses $[F(1,5)=0.002, p=0.97]$. Mcan reaction times for

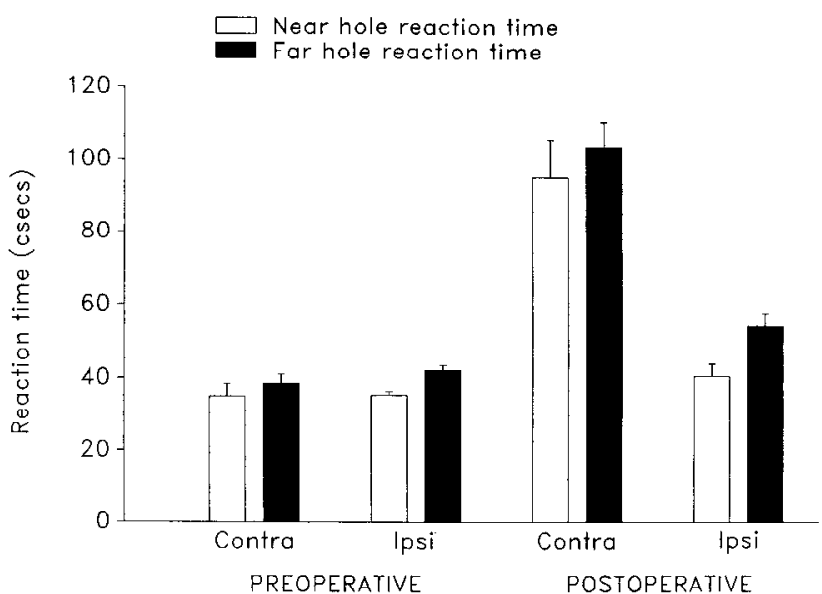

Figure 4. Reaction times of correct responses made in the near and far holes pre- and postoperatively for each group. The contralaterally lesioned animals had significantly lengthened reaction times to both stimuli, and the preoperative difference in reaction time of near and far hole responses was also no longer found. There was a nonsignificant increase in the reaction times of the ipsilaterally lesioned rats. 


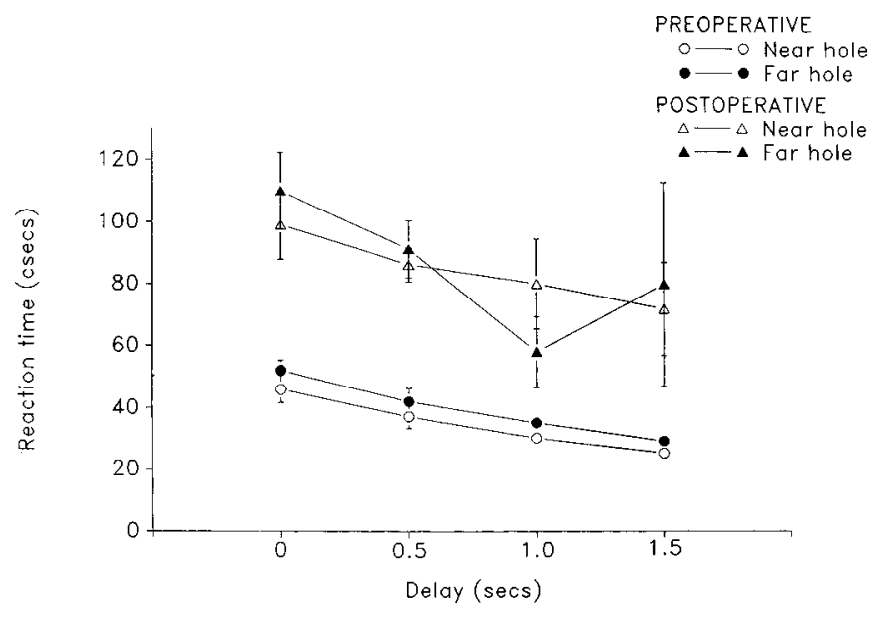

Figure 5. Reaction times of the contralaterally lesioned group plotted as a function of stimulus presentation delay. The increase in reaction times of the contralaterally lesioned rats was shown to be linear.

correct and incorrect responses in the baseline condition were 0.951 and $0.954 \mathrm{sec}$, respectively.

\section{Effects of omitting one of the stimuli}

Figure 6 shows reaction times in the baseline condition and for 2 sessions when first the stimulus in the near left hole and then the stimulus in the far left hole was removed in the ipsi- and contralaterally lesioned groups.

The 3-way interaction between the factors-side of Lesion, Condition (i.e., stimulus manipulation), and Hole-was significant $[F(2,17)=9.56, p<0.002]$. Analysis of the simple interaction of Condition and Hole for the ipsilaterally lesioned rats was also highly significant $[F(2,17)=46.6, p<0.002]$, which can be seen (Fig. 6, top) to result from the lengthened reaction time of responses in the locations from which the visual discriminanda were removed, with no change in reaction time to stimuli at the other location. Analysis of the Condition $\times$ Hole interaction for the contralaterally lesioned rats was compromised by the lack of sufficient data for that group in all of the conditions. Therefore, a single post hoc Student's $t$ test was applied to test the significance of the contrast between the reaction times of near hole responses for the baseline condition and the 2 stimulus manipulations, shown as open histogram blocks in Figure 6, bottom). This confirmed the significance of the effect that reaction times of responses made in the near hole became faster when either stimulus was removed $(t=2.16, p$ $<0.05$ ). As removing either the near or the far discriminanda altered reaction times (in both cases making them quicker), it can be concluded that the contralaterally lesioned rats must have been altending, to some extent, to both of the lights in the baseline condition, even though they made no correct responses to the far-left light.

\section{Effects of presenting both or neither stimuli}

Figure 7 shows the effect on postoperative bias of presenting neither, or both, of the stimuli to each of the lesion groups. The contralateral lesion group was biased under all stimulus conditions to the near hole. The ipsilateral lesion group was unbiased in the baseline condition and when neither stimulus was presented; they were biased only when both the stimuli were
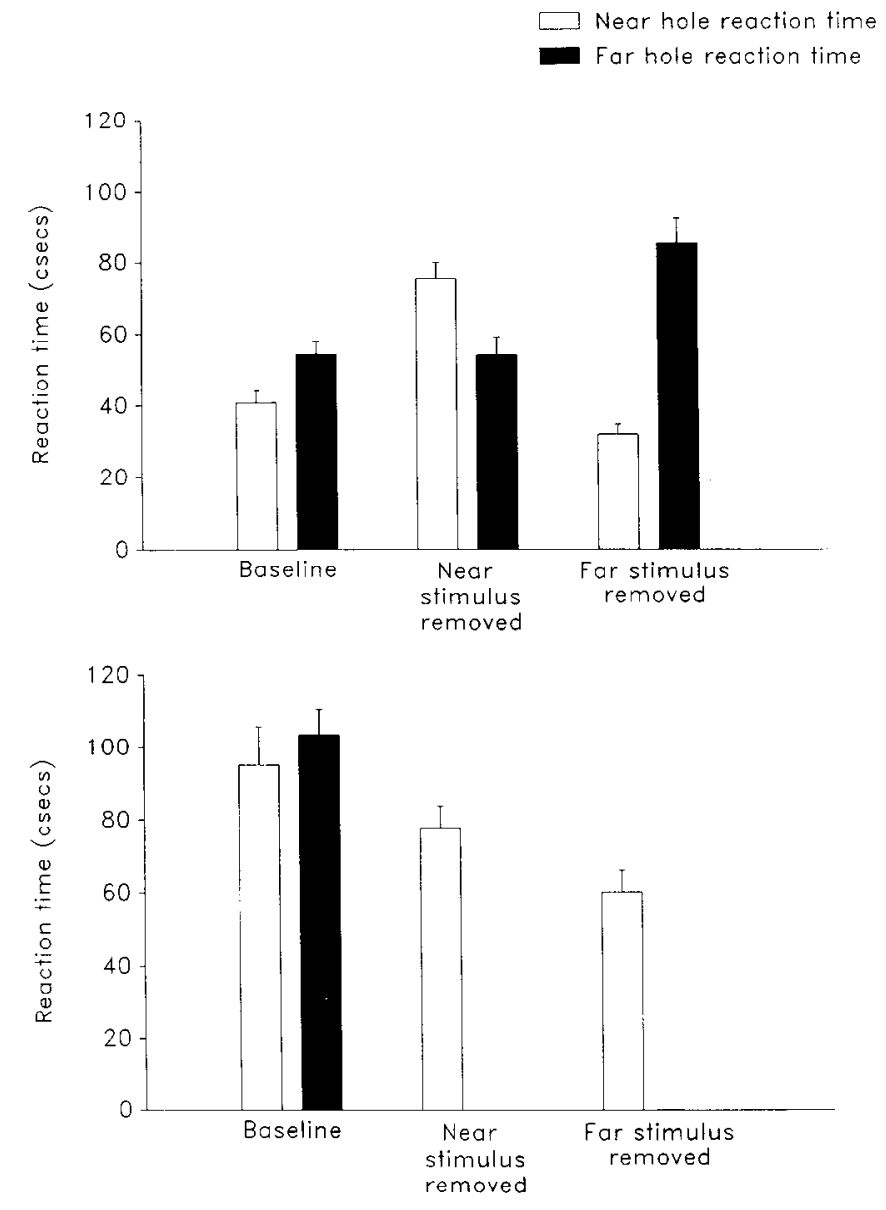

Figure 6. Reaction times in the baseline condition and when either one of the stimuli was removed for (top) ipsilaterally and (bottom) contralaterally lesioned animals. In the ipsilateral lesion group, reaction times of responses to a location were lengthened when that stimulus was removed; reaction time to the remaining stimulus was unaffected. In the contralateral lesion group, there were insufficient far hole responses to permit analysis, but reaction times of near hole responses were significantly speeded when either stimulus was removed.

presented simultaneously. This was reflected in a significant Lesion $\times$ Condition interaction $[F(2,26)=7.14, p=0.003]$.

\section{Effects of removing response options}

When the near response hole was blocked, the contralaterally lesioned rats were able to direct responses to the far hole, demonstrating that a simple motor impairment was not the cause of the response bias to the near hole. Figure 8 shows the speeding of reaction times of the contralaterally lesioned rats to the near and the far stimuli in the simple reaction time task compared with baseline performance. In a comparison of baseline and simple reaction time performance, significant interactions between the factors side of Lesion and Condition $[F(1,13)=13.02$, $p=0.003]$ and Lesion and Hole $[F(1,25)=5.9, p=0.02]$ were found. Simple main effect analyses showed that (1) Condition was significant in the contralateral, but not the ipsilateral, lesion group $[F(1,13)=38.6, p<0.001]$, and (2) Hole was significant in the ipsilateral but not contralateral lesion group $[F(1,25)=$ $11.2, p<0.001]$. That is, for the contralateral lesion group, simple reaction time was faster than choice reaction time, while for the ipsilateral lesion group, responses in the near hole were faster than responses in the far hole. 


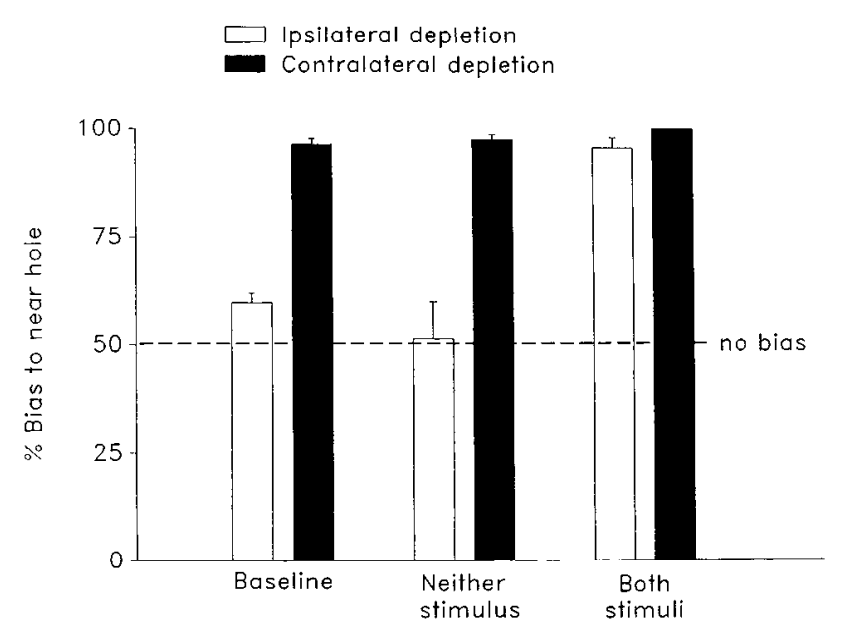

Figure 7. Near hole response bias in the baseline condition and when neither or both stimuli were presented. The ipsilaterally lesioned rats were significantly biased only when both stimuli were presented simultaneously; seeing no stimulus resulted in random responses in either hole, with an equal number of responses in each. The contralaterally lesioned animals were biased to the near hole in all conditions.

\section{Lack of behavioral recovery}

The rats were tested again 4 weeks after surgery for 5 consecutive days. There was no evidence of behavioral recovery in the contralaterally lesioned rats; the bias of $96 \%$ seen in the first postoperative baseline was nonsignificantly increased to $99 \%$ by the second period of postoperative testing $(t=0.6, p>0.05)$.

\section{Discussion}

The results presented here have shown that following unilateral striatal DA depletion, there was (1) a response bias to the nearer of 2 holes in contralateral space; (2) a lengthening of reaction times when a response was made in contralateral space; (3) no significant impairment in rats lesioned ipsilateral to the side of performance.

Other authors have found that similarly induced neglect often recovers within a few weeks when conventionally assessed using observer ratings (Marshall, 1979); the sensitivity of the measures used in this experiment enabled us to quantify long-lasting behavioral effects, and we found no evidence of recovery up to 35 $\mathrm{d}$ after the lesion.

We are interpreting the behavioral results in terms of the effects of relatively selective damage inflicted by the neurotoxin 6-OIIDA. The selectivity of this agent has been previously discussed (Breese and Traylor, 1971), and our discussion therefore focuses on the nature of the behavioral impairment produced by unilateral striatal DA depletion and its significance for theories of striatal function.

The rats in this experiment did not have primary motor impairment; they were still able to initiate trials by making sustained nose pokes to deliver stimuli and were also able to make movements in contralateral space. During the periods of timeout, following incorrect or inappropriate responses, responses were directed to both the near and the far holes, and, in addition, all rats could respond in the far hole when the near hole was blocked in a simple reaction time task. These observations further indicate that the rats had no deficit in the retention of the task requirements.

There was significant depletion of DA from the tail of the

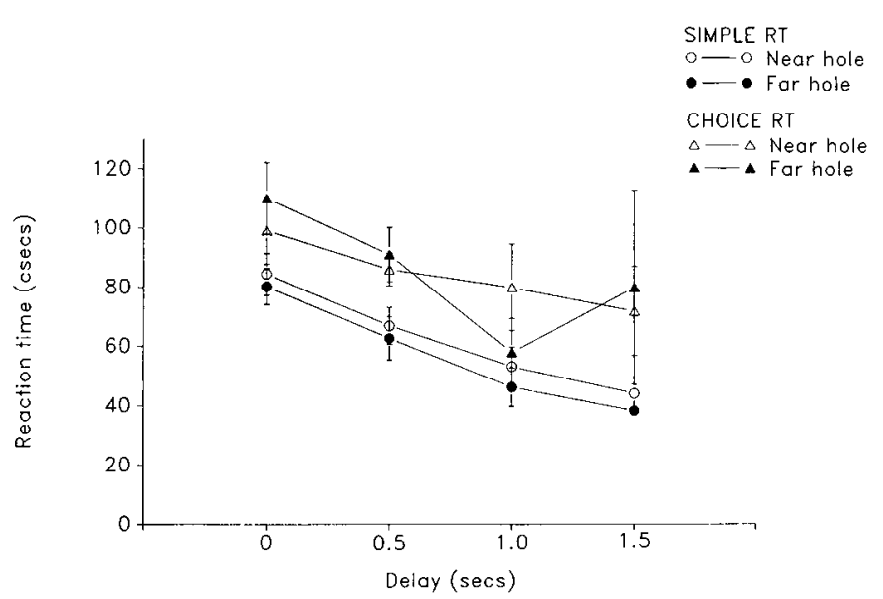

Figure 8. Choice and simple reaction times of the contralaterally lesioned animals plotted as a function of delay. Reaction time was significantly speeded when either the near or the far hole was blocked and stimuli were presented in one hole only, in a test of simple reaction time.

caudate, and in view of the projections to this region from visual cortical areas in primates (Kemp and Powell, 1970) and the previously reported impairments on visual discrimination following lesions of the tail of the caudate in the monkey (Divac et al., 1967), the possibility of visual impairment was considered. However, there are several lines of evidence to suggest that the response bias and reaction time effects were not due to primary sensory deficits. First, it is clear that the contralateral group was under a degree of stimulus control. For example, the reaction time data suggest that the rats were not simply waiting for a suitable period (i.e., the longest stimulus delay) to elapse before responding, because there was an equivalent lengthening of reaction time at all stimulus delays (see also Carli et al., 1989). There were also significant reductions in reaction time to the remaining stimulus in the sessions in which one of the stimulus lights was removed. As removing either the near or far left stimulus had an effect on performance, we can infer that the rat was able to detcct the stimulus to some extent when it was present. Most convincing, however, is the fact that in probe trials when neither stimulus was presented, the control, ipsilaterally lesioned rats were not biased in their responses. Thus, unlike the effect of depleting striatal DA contralaterally, seeing neither stimulus did not result in a bias to the nearer of the 2 holes. Therefore, a failure to detect contralateral visual stimuli can account for neither the enhanced response bias nor the reaction time effect, and we conclude that the lesions did not result in a primary sensory deficit.

The contralaterally lesioned rats may have been able to detect both stimuli but unable to localize them accurately in contralateral space, a form of alloaesthesia (Obersteiner, 1882), but this hypothesis cannot account for all aspects of the behavior. There is no reason why alloaesthesia should have resulted in a bias to the near hole, as opposed to a general decline in accuracy. If the animal had been unable to localize stimuli in contralateral space, they should have been equally impaired in localizing near and far stimuli. Furthermore, alloaesthesia cannot account for the deficit found in trials when no stimuli were presented. When the ipsilaterally lesioned rats are presented with no stimulus, they respond in either hole and are not biased; if the contralaterally lesioned animals are impaired only in localizing stimuli, 
they should show no impairment when there are no stimuli. As the bias was found irrespective of the configuration, or even presence, of the stimulus, it must arise from a response-related deficit, and it is therefore concluded that the deficits did not stem from an inability to localize the stimuli.

Rather than the stimuli being mislocated, the evidence suggests that the responses were being misdirected. Finding no reaction time difference between correct and incorrect responses supports the view that the rats were discriminating the stimuli with equal facility, and were responding with equal confidence to both, but were misdirecting their responses towards the near hole. When one of the response locations was blocked, in a simple reaction time task, the contralaterally lesioned rats were able to make responses to both the near and the far response locations in turn, and in the absence of response alternatives, reaction times were significantly speeded, possibly due to responses being made with increased confidence when the possibility of being incorrect was removed.

It is proposed that the inability to direct responses into contralateral space is due to a disruption of the spatial coding of responses or of the representation of space into which responses are made, with responses no longer differentially coded in spatial coordinates.

The design of this experiment allowed a test of 2 alternative hypotheses concerning the form of a spatial impairment. First, neglect resulting from striatal DA depletion is restricted to one side of the body, or alternatively, that it is relative, or allocentric, in nature. The data presented here suggest that unilateral striatal lesions result in an egocentric type of neglect, in which the response options are spatially coded not in terms of relative contralateral position but, rather, in terms of absolute contralateral space. The contralaterally lesioned animals were less impaired in responding to the near, or relatively ipsilateral, hole, which could be due to proximal, or shorter, movements, being differentially affected compared with more distal, or longer, movements (Hallett and Khoshbin, 1980; Whishaw and Dunnett, 1985; Weinrich et al., 1988) or simply because responses initiated towards the stimuli reach the nearer of the 2 holes first. When the near hole was blocked, they were capable of initiating far hole responses with equivalent speed and accuracy as for near hole responses. The ipsilaterally lesioned animals were not impaired in their accuracy of performance of the task, nor were they slower to initiate responses to the near (for them, relatively contralateral) hole. Thus, it is concluded that the rats in this experiment did not show a neglect for stimuli in a relatively contralateral position.

In conclusion, novel insights have been obtained into the nature of "striatal neglect." The data presented here support the view that a response impairment is the primary deficit and apparent sensory-attentional changes arise from, and are secondary to, this impairment. From the results of the present experiments the previously reported deficits in initiating responses to contralateral space can be understood as resulting from a distortion of the space into which responses are directed. The nature of the striatal representation of response space is held to be primarily egocentric, rather than allocentric, in character, which is in agreement with previous findings (Potegal, 1969; Abraham et al., 1983; Hikosaka and Wurtz, 1985; Cook and Kesner, 1988). This hypothesis might help to account for the well-known impairment in Parkinson's disease in which paticnts are unable to dircet movements into spacc casily unless that space is given "structure" by the provision of visual stimuli such as lines drawn on the floor (Martin, 1967).

\section{References}

Abraham, L., M. Potegal, and S. Miller (1983) Evidence for caudate nucleus involvement in an egocentric spatial task: Return from passive transport. Physiol. Psychol. 11: 11-17.

Amalric, M., and G. F. Koob (1987) Depletion of dopamine in the caudate nucleus but not in nucleus accumbens impairs reaction time performance in rats. J. Neurosci. 7: $2129-2134$.

Bender, M. B. (1952) Disorders of Perception, Thomas, Springfield, IL.

Bisiach, E., and C. Luzzatti (1978) Unilateral neglect of representational space. Cortex 14: 129-133.

Breese, G. R., and T. D. Traylor (1971) Depletion of brain noradrenaline and dopamine by 6 -hydroxydopamine. Br. J. Pharmacol. 42. 88-99.

Carli, M., T. W. Robbins, J. L. Evenden, and B. J. Everitt (1983) Effects of lesions to ascending noradrenergic neurons on performance of a five-choice serial reaction task in rats; implications for theories of dorsal noradrenergic function based on selective attention and arousal. Behav. Brain Res. 35: 443-457.

Carli, M., J. L. Evenden, and T. W. Robbins (1985) Depletion of unilateral striatal dopamine impairs initiation of contralateral actions and not sensory attention. Nature 313: 679-682.

Carli, M., G. H. Jones, and T. W. Robbins (1989) Effects of unilateral dorsal and ventral striatal dopamine depletion on visual neglect in the rat: A neural and behavioral analysis. Neuroscience (in press).

Cook, D., and R. P. Kesner (1988) Caudate nucleus and memory for egocentric localization. Behav. Neural Biol. 49: 332-343.

Cowey, A., and C. Francini (1979) The retinal origin of uncrossed optic nerve fibers in rats and their role in visual discrimination. Exp. Brain Res. 35: 443-457.

Divac, I., H. E. Rosvold, and M. K. Szwarcbart (1967) Behavioral effects of selective ablation of the caudate nucleus. J. Comp. Physiol. Psychol. 63: 184-190.

Dunnett, S. B., and S. D. Iversen (1982) Sensorimotor impairments following localized kainic acid and 6-OHDA lesions of the neostriatum. Brain Res. 248: 121-127.

Fairley, P. C., and J. F. Marshall (1986) Dopamine in the lateral caudate-putamen of the rat is essential for somatosensory orientation. Behav. Neurosci. 100: 652-663.

Hallett, M., and S. Khoshbin (1980) A physiological mechanism of bradykinesia. Brain 103: 301-314.

Hikosaka, O., and R. H. Wurtz (1985) Modification of saccadic eye movements by GABA related substances: II. Effects of muscimol in monkey substantia nigra pars reticulata. J. Neurophysiol. 53: 292308 .

Kemp, J. M., and T. P. S. Powell (1970) The cortico-striate projection in the monkey. Brain 93: 525-546.

Kinsbourne, M., and E. K. Warrington (1962) A variety of reading disability associated with right hemisphere lesions. J. Neurol. Neurosurg. Psychiatry 25: 339-334.

Koob, G. F., S. J. Riley, S. C. Smith, and T. W. Robbins (1978) Effects of 6-hydroxydopamine lesions of the nucleus accumbens or olfactory tubercle on feeding, locomotor activity, and amphetamine anorexia in the rat. J. Comp. Physiol. Psychol. 92: 917-927.

Ladavas, E. (1987) Is the hemispatial deficit produced by right parietal lobe damage associated with retinal or gravitational coordinates? Brain 110: $167-180$.

Ljungberg, T., and U. Ungerstedt (1976) Sensory inattention produced by 6-OHDA-induced degeneration of ascending DA neurons in the brain. Exp. Neurol. 53: 585-609.

Marshall, J. F. (1979) Somatosensory inattention after dopaminedepleting intracerebral 6-OHDA injections: Spontaneous recovery and pharmacological control. Brain Res. 177: 311-324.

Marshall, J. F., and P. Teitelbaum (1974) Sensory neglect produced by lateral hypothalamic damage in rats. J. Comp. Physiol. Psychol. 86: 375-395.

Marshall, J. F., B. H. Turner, and P. Teitelbaum (1971) Sensory neglect produced by lateral hypothalamic damage. Science 174: 523-525.

Marshall, J. F., N. G. Berrios, and S. Sawyer (1980) Neostriatal do- 
pamine and sensory inattention. J. Comp. Physiol. Psychol. 94: 833846.

Martin, J. P. (1967) The Basal Ganglia and Posture, Pitman Medical, London.

Mefford, I. N. (1981) Application of high performance liquid chromatography with electrochemical detection to neurochemical analysis: Measurement of catecholamines, serotonin and metabolites in the rat brain. J. Neurosci. Methods 3: 310-338.

Mittleman, G. M., I. Q. Whishaw, and T. W. Robbins (1988) Cortical lateralization of function in rats in a visual reaction time task. Behav. Brain Res. 31: 29-36.

Obersteiner, H. (1882) On allochiria-a peculiar sensory disorder. Brain 4: 153-163.
Pellegrino, L. J., A. S. Pellegrino, and A. I. Cushman (1979) A Stereotaxic Atlas of the Rat Brain, 2nd ed., Plenum, New York.

Potegal, M. (1969) Role of the caudate nucleus in spatial orientation in rats. J. Comp. Physiol. Psychol. 69: 756-764.

Weinrich, M., K. Koch, F. Garcia, and R. W. Angel (1988) Axial versus distal motor impairment in Parkinson's disease. Neurology 38: 540-545.

Whishaw, I. Q., and S. B. Dunnett (1985) Dopamine depletion, stimulation or blockade in the rat disrupts spatial navigation and locomotion dependent upon beacon or distal cues. Behav. Brain Res. 18: 11-29.

Winer, B. J. (1971) Statistical Principles in Experimental Design, McGraw-Hill, New York. 